Search for a Single-Photon Anomalous Excess in MicroBooNE

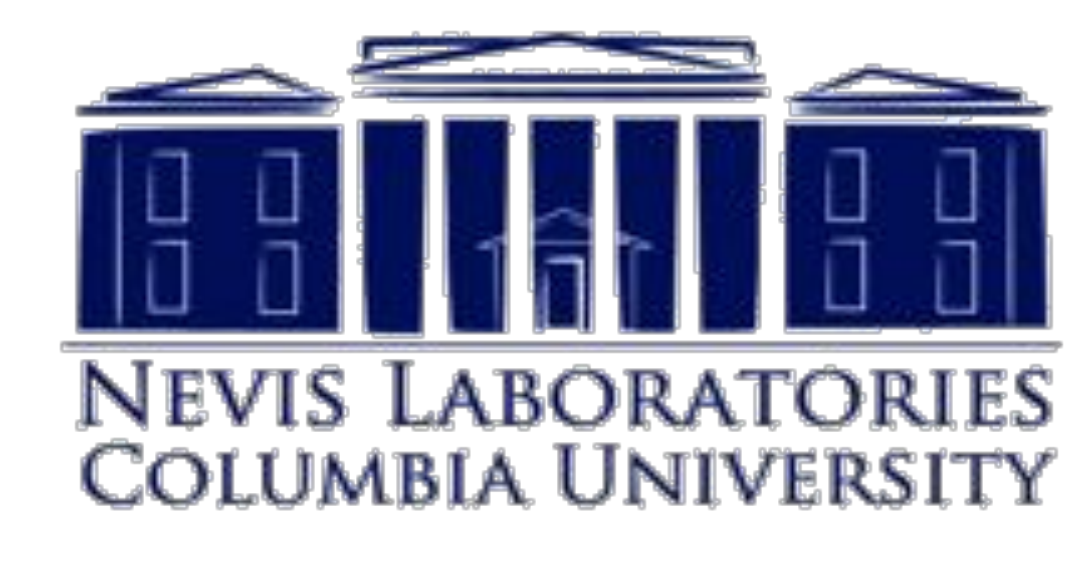

1. The "Low Energy Excess (LEE)"

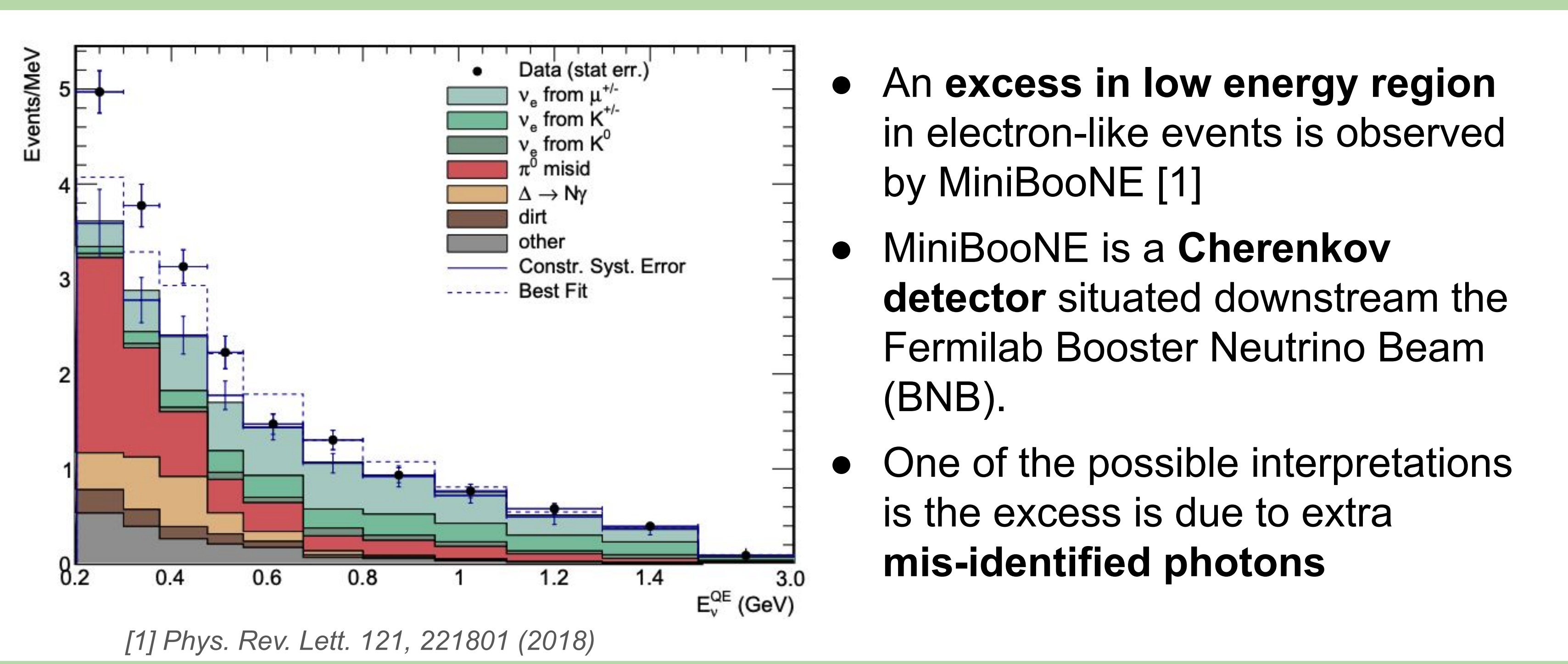

4. Final Distributions
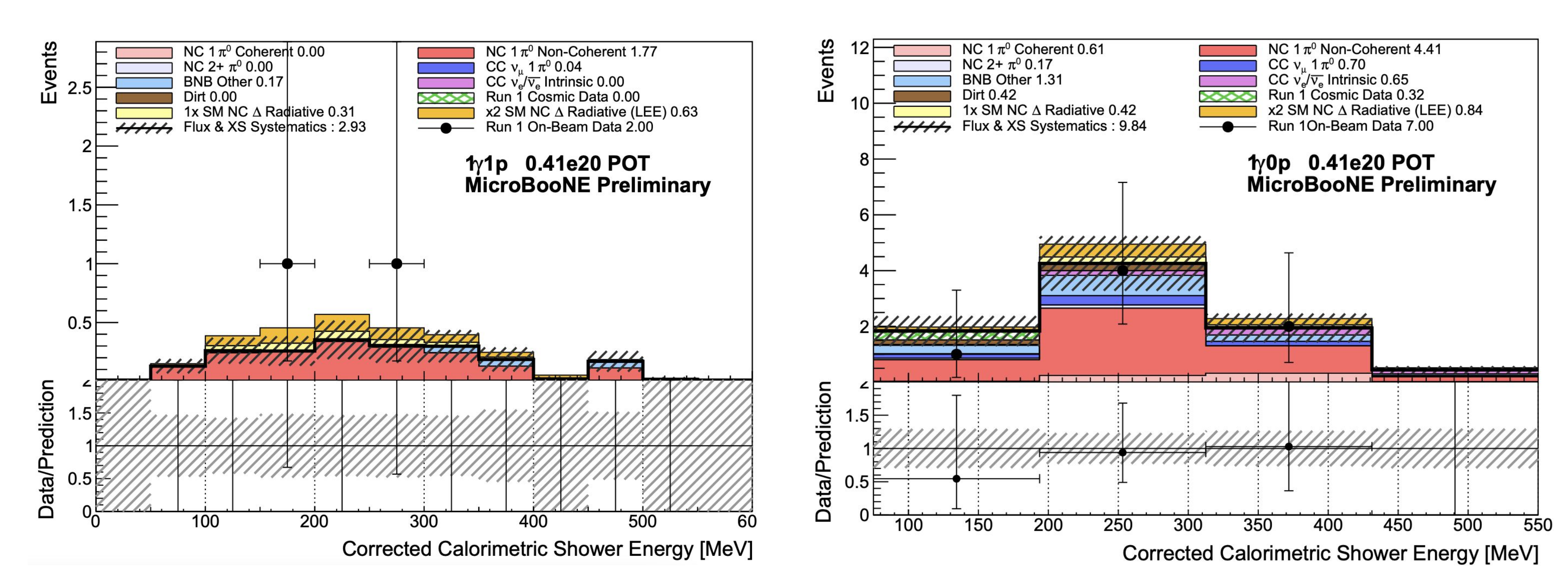

- Overall good agreement between data and $\mathrm{MC}$ central value (CV) for both selection

- Due to blind analysis policy, the final distributions here are from only $5 \%$ of the full

MicroBooNE Run1-5 data set, but $\sim 15 x$ more statistics are expected once Run1-3 data

is unblinded

- Note that $\mathrm{NC} \pi^{0}$ is the dominant background in both selections

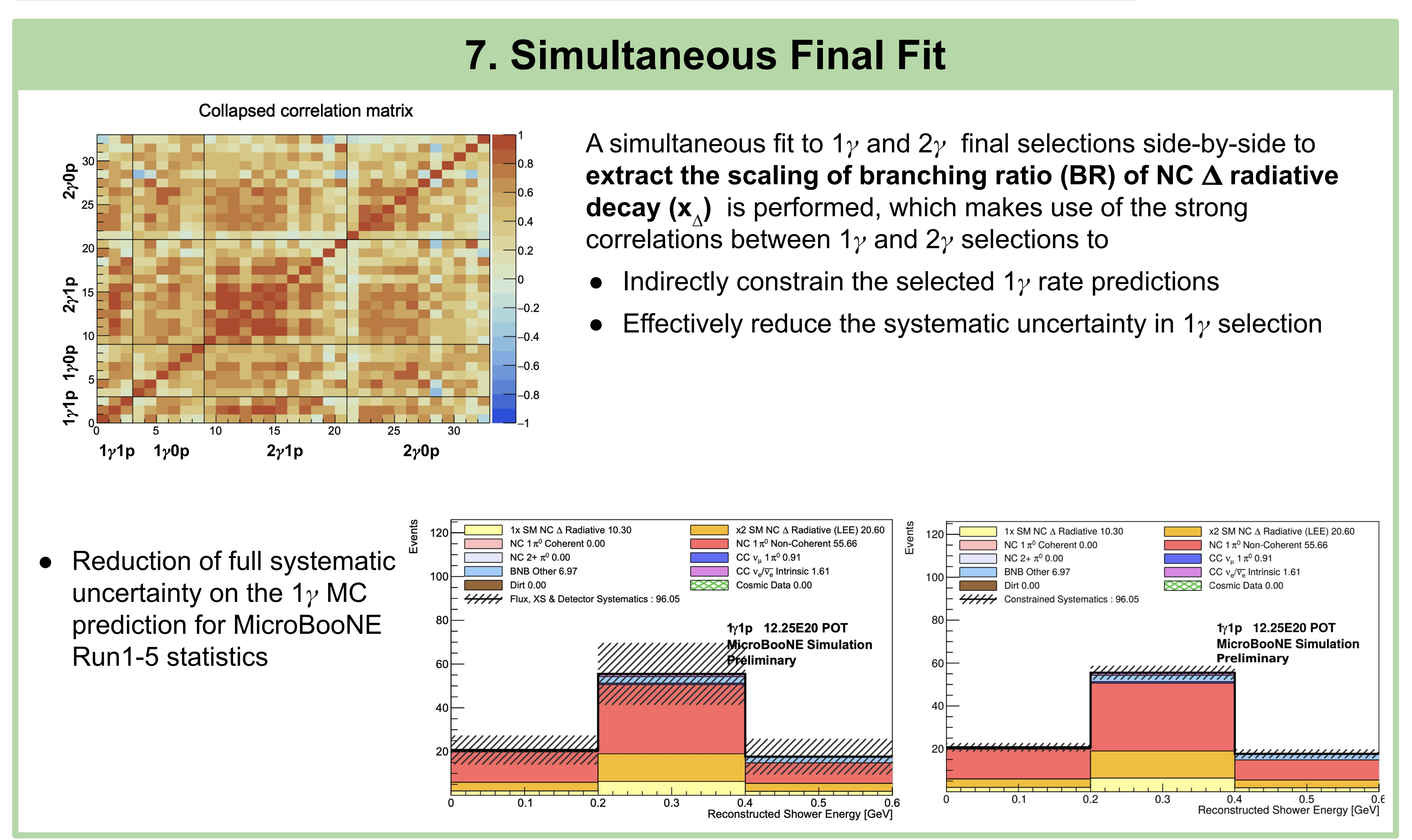

\section{Guanqun Ge}

Columbia University

representing the MicroBooNE collaboration
MBOoNE

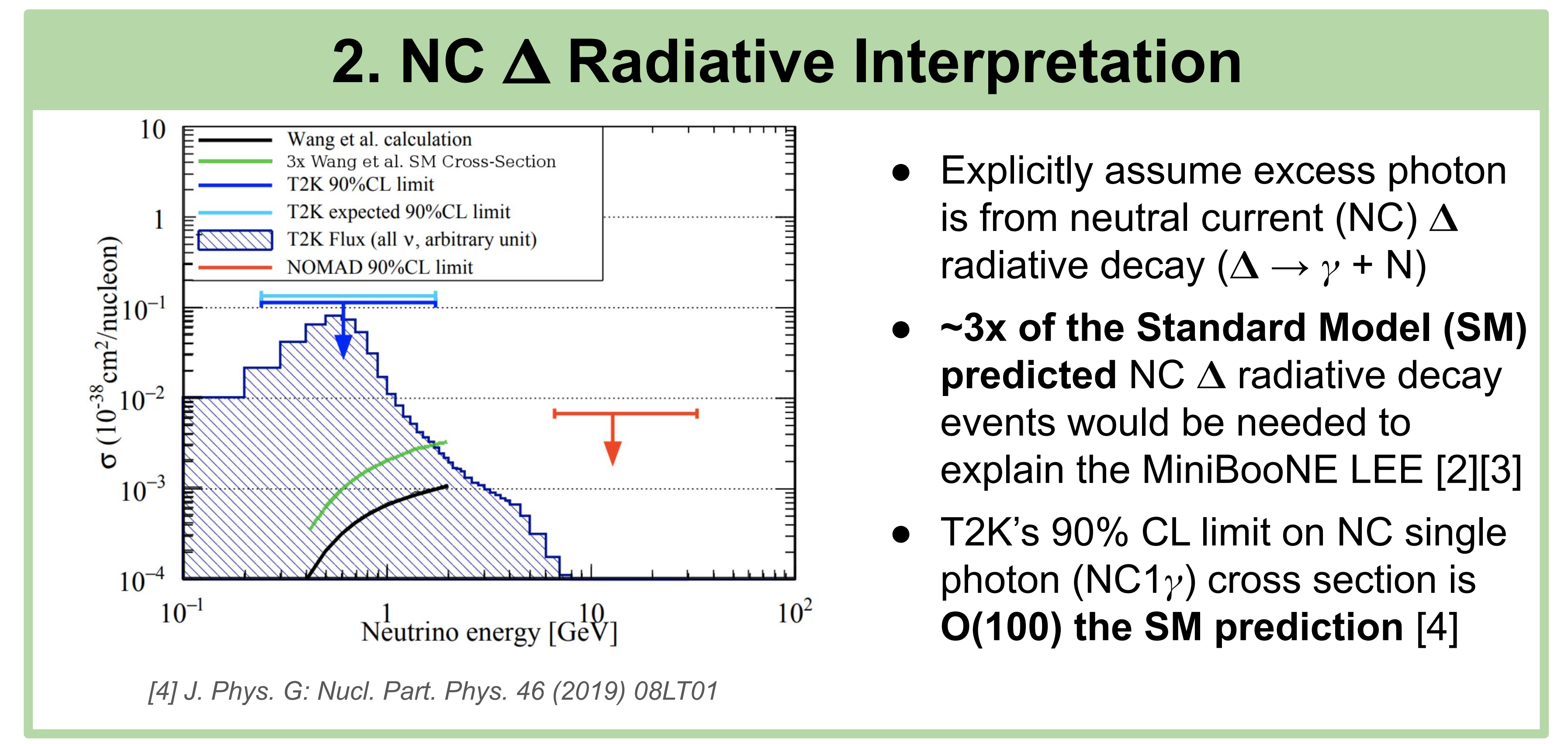

5. NC $\pi^{0}$ Measurement

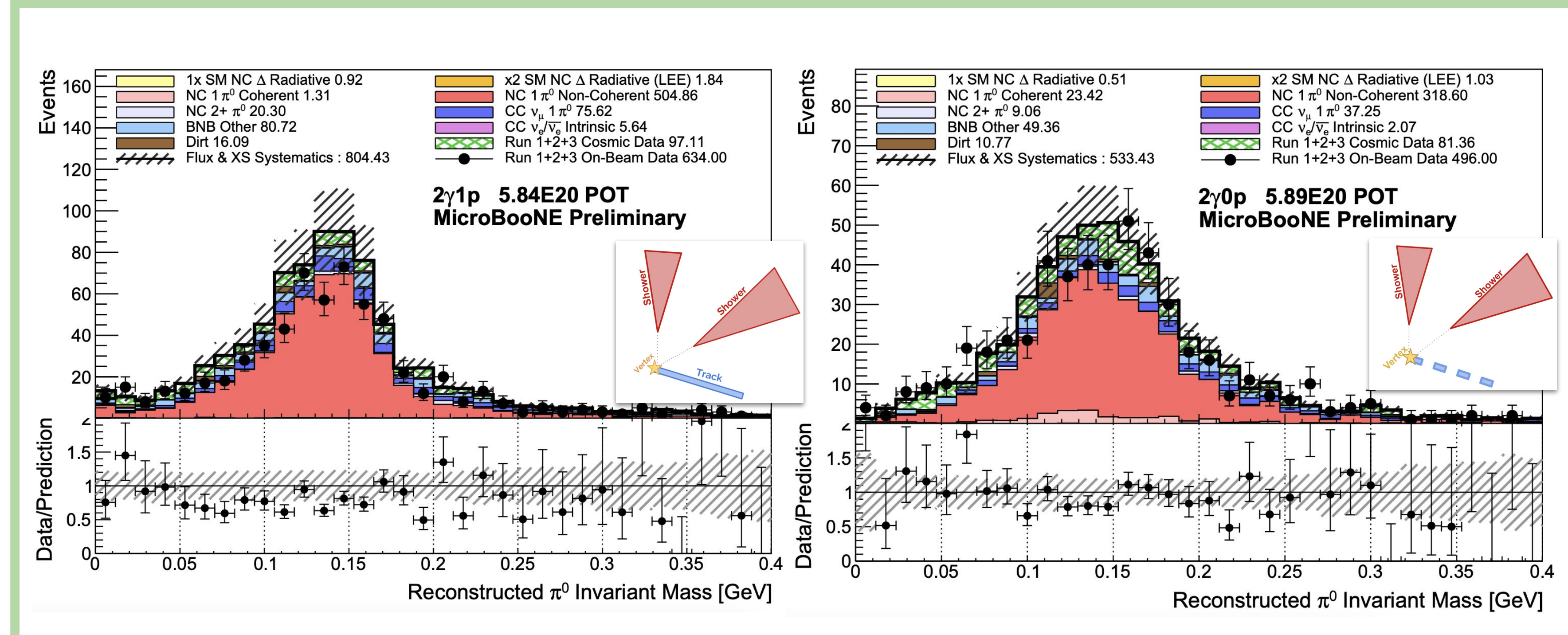

- High-statistics measurement, high purity of $\mathrm{NC} \pi^{0}(\sim 60 \%)$ in both selections - With flux and cross section uncertainty, data and MC CV agree reasonably well

$\sim 20 \%$ and $\sim 7 \%$ data deficit observed in $2 \gamma 1 p$ and $2 \gamma 0$ p respectively

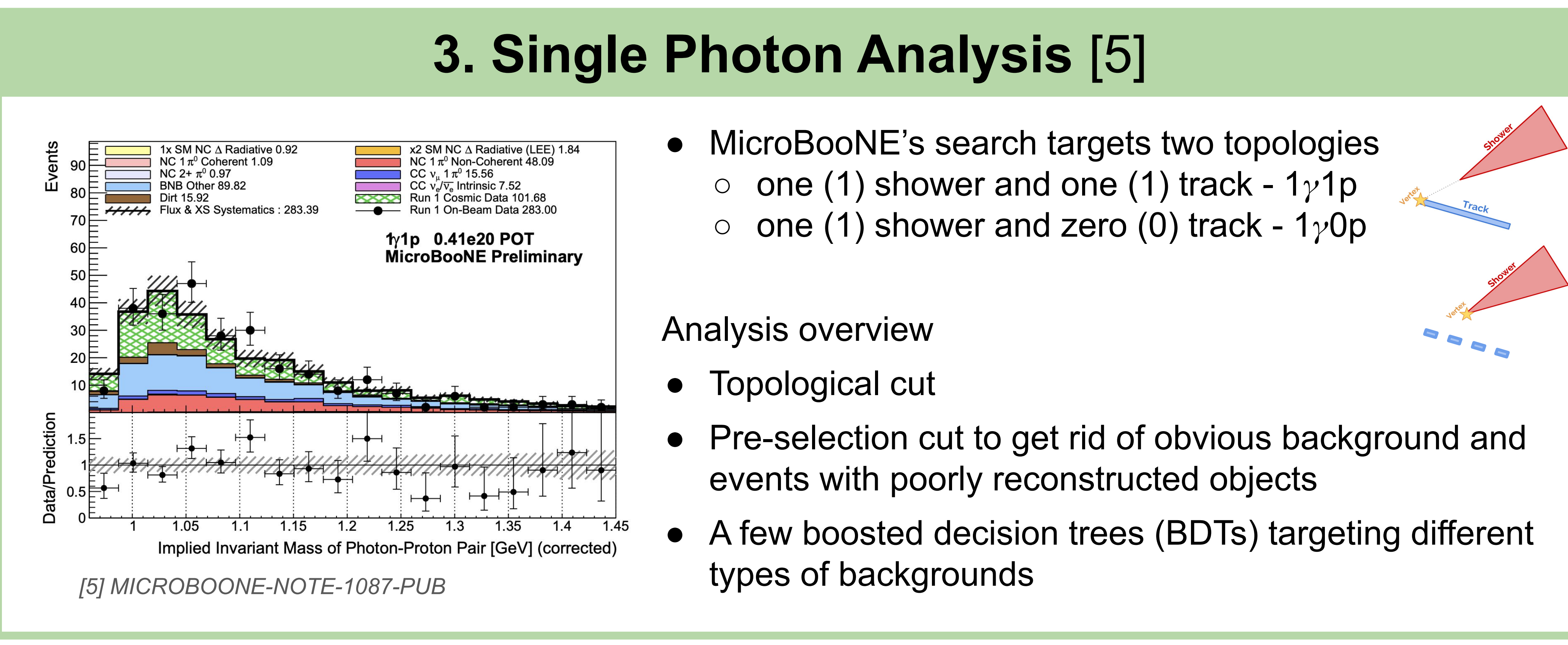

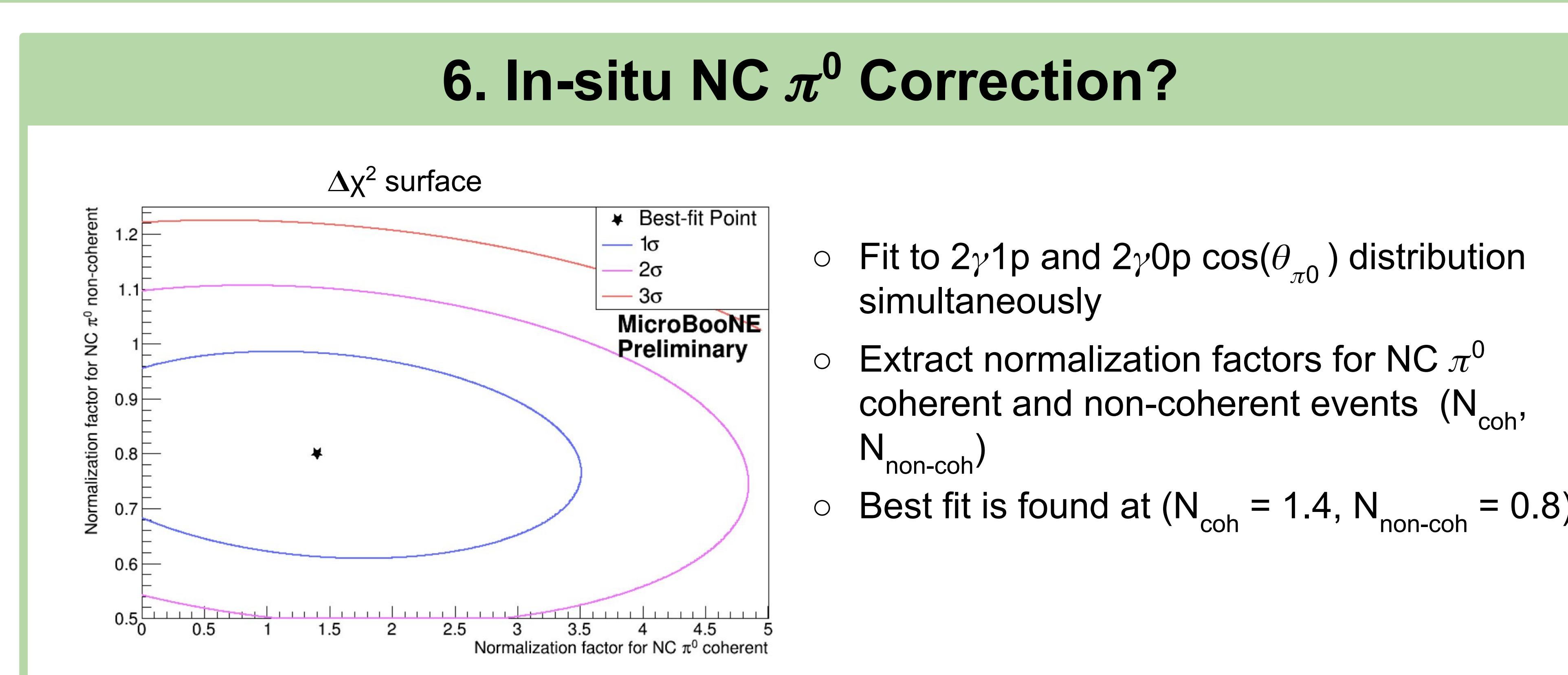

- While $M C C V\left(N_{c o b}=1, N_{n o n-c o n}=1\right)$ sits outside the $1 \sigma$ region of the data-derived uncertainty, data is consistent with MC CV given large GENIE uncertainty - Instead of correcting GENIE prediction of NC $\pi^{0}$, simultaneous fit of both $1 \gamma$ and $2 \gamma$ selections is performed to constrain background in $1 \gamma$ selection

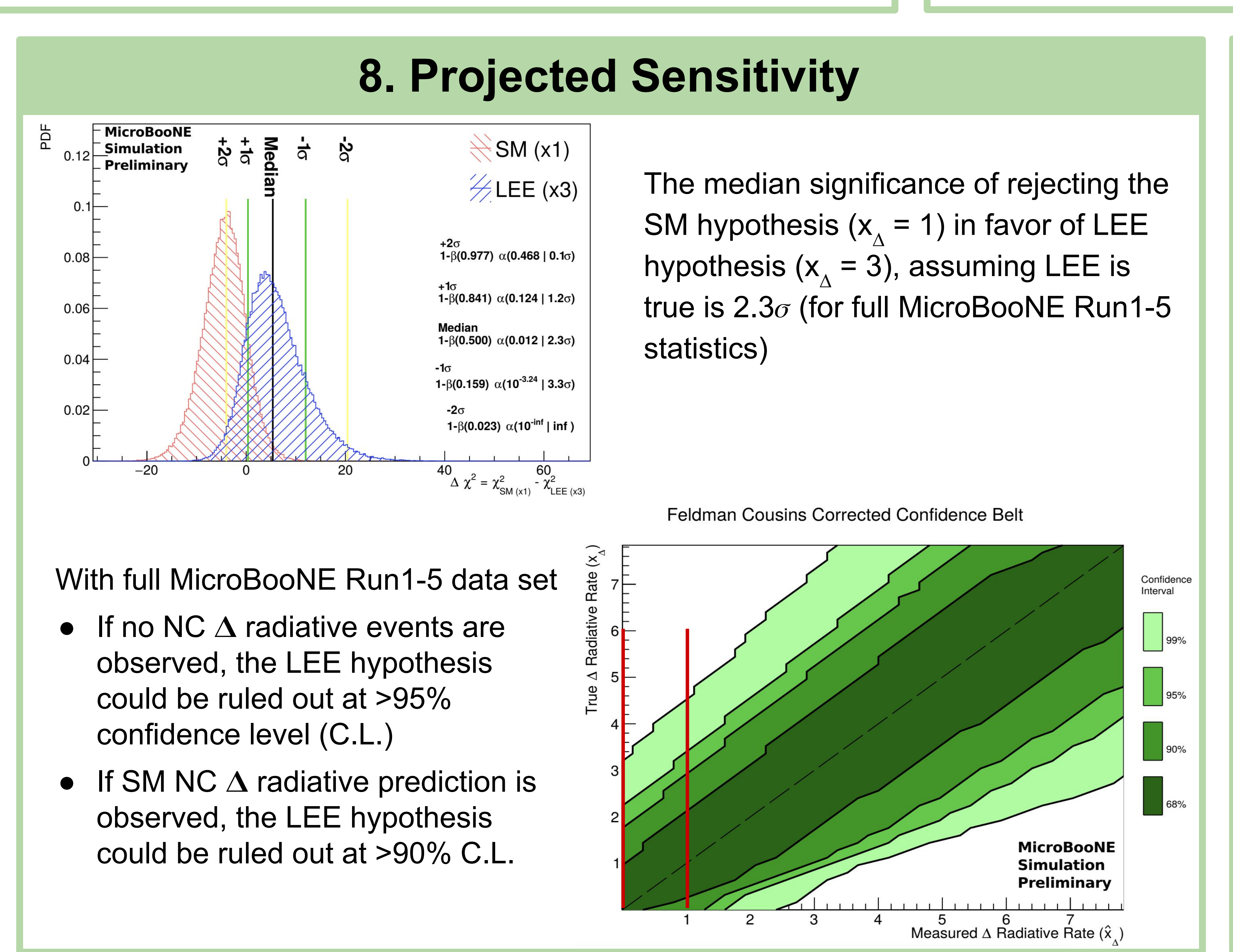

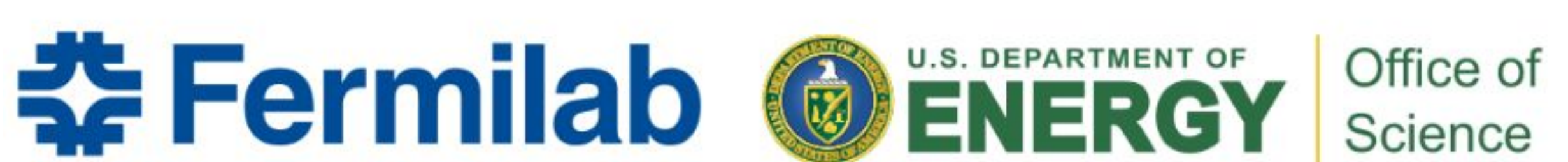

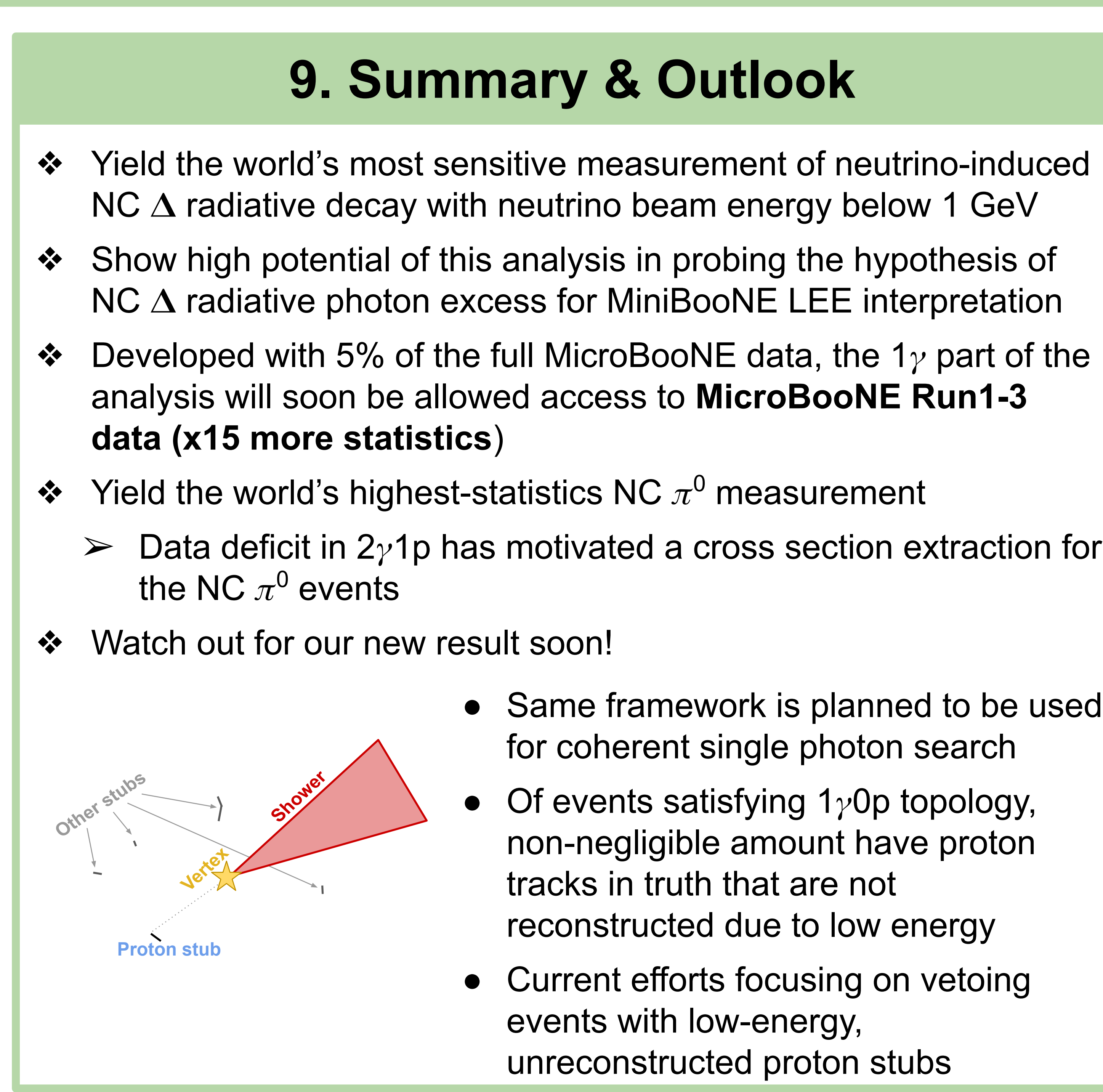

FNSNF unreconstructed proton stubs 